University of Nebraska - Lincoln

DigitalCommons@University of Nebraska - Lincoln

November 1991

\title{
Development of the $\mathrm{Gd}(0001)$ band structure with film thickness
}

Dongqi Li

Syracuse University

W. Hutchings

Syracuse University

Peter A. Dowben

University of Nebraska-Lincoln, pdowben@unl.edu

Rong-Tzong Wu

University of Wisconsin, Madison

C. Hwang

University of Wisconsin, Madison

See next page for additional authors

Follow this and additional works at: https://digitalcommons.unl.edu/physicsdowben

Part of the Physics Commons

Li, Dongqi; Hutchings, W.; Dowben, Peter A.; Wu, Rong-Tzong; Hwang, C.; Onellion, M.; Andrews, A.B.; and Erskine, J.L., "Development of the Gd(0001) band structure with film thickness" (1991). Peter Dowben Publications. 101.

https://digitalcommons.unl.edu/physicsdowben/101

This Article is brought to you for free and open access by the Research Papers in Physics and Astronomy at DigitalCommons@University of Nebraska - Lincoln. It has been accepted for inclusion in Peter Dowben Publications by an authorized administrator of DigitalCommons@University of Nebraska - Lincoln. 


\section{Authors}

Dongqi Li, W. Hutchings, Peter A. Dowben, Rong-Tzong Wu, C. Hwang, M. Onellion, A.B. Andrews, and J.L. Erskine 


\title{
Development of the $\mathrm{Gd}(0001)$ band structure with film thickness
}

\author{
Dongqi Li, C. W. Hutchings, ${ }^{\text {a) }}$ and P. A. Dowben ${ }^{\text {b) }}$
}

Department of Physics, Syracuse University, Syracuse, New York 13244-1130

Rong-Tzong Wu, C. Hwang, ${ }^{c)}$ and M. Onellion

Department of Physics, University of Wisconsin, Madison, Wisconsin 53706

\author{
A. B. Andrews and J. L. Erskine \\ Department of Physics, University of Texas, Austin, Texas 78712
}

By growing gadolinium films on W(110), it has been possible to observe the development of
the Gd(0001) band structure with increasing film thickness. The changes in the $5 d$
bands as a result of exposure to oxygen or the development of the valence-band structure
with increasing film thickness lead to changes in the $5 p$ multiplet oscillator strengths.
These changes are a result of the influence of the band structure upon the final-state effects
that give rise to such multiplet structures. This study provides conclusive evidence
that there is a $5 d$ contribution to the shallow $5 p$ multiplet photoemission core-level structure.

\section{INTRODUCTION}

Both theoretical ${ }^{1-9}$ and experimental $1^{10,11}$ studies of the gadolinium band structure have been undertaken. Recently, a Gd(0001) magnetic surface state has been predicted ${ }^{9}$ and observed. ${ }^{10}$ In this paper we show that this surface state and the bulk band structure can only be distinguished for the relatively thicker films as a result of the development of the Gd(0001) band structure. Because the $5 d$ bands play a significant role in photoemission from the $5 p$ shallow core levels as a result of the final-state coupling, ${ }^{11-15}$ the development of the $5 d$ band structure is expected to affect the $5 p$ multiplets. Changes in the $5 p$ multiplet structure with increasing film thickness can indeed be observed as are expected.

By depositing gadolinium on W(110), the development of the gadolinium band structure with increasing film thickness can be characterized. Films deposited on $\mathrm{W}(110)$ have been studied by a number of groups ${ }^{16-24}$ and several studies have established that the films grow and adopt the crystal structure of bulk gadolinium with the (0001) surface parallel with the $\mathrm{W}(110)$ surface (i.e., the $c$ axis grows along the surface normal). ${ }^{16-18}$ This property of the gadolinium films grown on W(110) makes this rareearth overlayer system particularly useful for investigating the development of the electronic band structure with increasing film thickness for crystalline gadolinium without the contamination problems common to the rare earths. ${ }^{11,25,26}$

\section{EXPERIMENT}

The gadolinium was deposited on clean W(110) as has been described previously for gadolinium deposition onto other substrates. ${ }^{12}$ The crystallinity and quality of the films deposited on $\mathrm{W}(110)$ was carefully characterized by low-

\footnotetext{
a) Present address: Institut fuer Angewandte Physikalische Chemie der Universität Heidelberg, Im Neuenheimerfeld 253, D-6900, Heidelberg, Germany.

b) Address all correspondence to this author.

c) Present address: Korean Energy Institute, Dae Jeon, Science City, Korea.
}

energy electron diffraction (LEED). The thickness of the gadolinium films was ascertained from a quartz-crystal oscillator thin-film monitor (Leybold-Inficon) but the film thicknesses cited in this work are approximate, as noted elsewhere. ${ }^{12}$ All binding energies cited in this work are referenced to the Fermi energy of the clean W(110) surface. Throughout this work, the light incidence angle was $70^{\circ}$ so that the vector potential is largely normal to the surface ( $p$-polarized light) unless otherwise noted. In this geometry, the surface state is greatly enhanced as discussed in detail elsewhere. ${ }^{10}$ The photoelectrons were collected normal to the surface (i.e., $\mathbf{k}_{11}=0$ or $\bar{\Gamma}^{\prime}$ ) into hemispherical electron energy analyzers described elsewhere, ${ }^{12,27}$ with angular resolutions of $\pm 1.5^{\circ}$. The light sources for this photoemission study were the NSLS vuv ring and the 1-GeV (Alladin) ring at the Synchrotron Radiation Center with the light dispersed in each case by a toroidal grating monochromator. The combined energy resolution (analyzer and monochromator) of the photoemission spectra varied from 0.15 to $0.2 \mathrm{eV}$ full width at half-maximum for the photoenergies taken between 30 and $50 \mathrm{eV}$.

\section{RESULTS AND DISCUSSION}

With increasing amounts of gadolinium deposited upon W(110), the gadolinium features quickly become the most prominent features in the energy distribution curves as seen in Fig. 1. For the very thin gadolinium films, there are two prominent features: the $4 f$ level at a binding energy of $8.6 \mathrm{eV}$ and the $5 d$ bands near the Fermi energy. For photoemission in the normal diraction $\left(\mathbf{k}_{11}=0\right.$ or $\left.\Gamma^{\prime}\right)$, the gadolinium bands become resolved into at least two distinct features with increasing coverage as seen in Fig. 1. With a $30-\AA$-thick gadolinium film, the feature near the Fermi energy has become quite narrow with a half-width of $300-400 \mathrm{meV}$. There is another $5 d$ band feature at a somewhat larger binding energy of $1-2 \mathrm{eV}$ binding energy. This feature becomes increasingly intense with increasing gadolinium film thickness. This higher-binding-energy feature has a binding energy of $1.5 \mathrm{eV}$ by $30 \AA$, but has a half-width of some $800 \mathrm{meV}$ or more. 


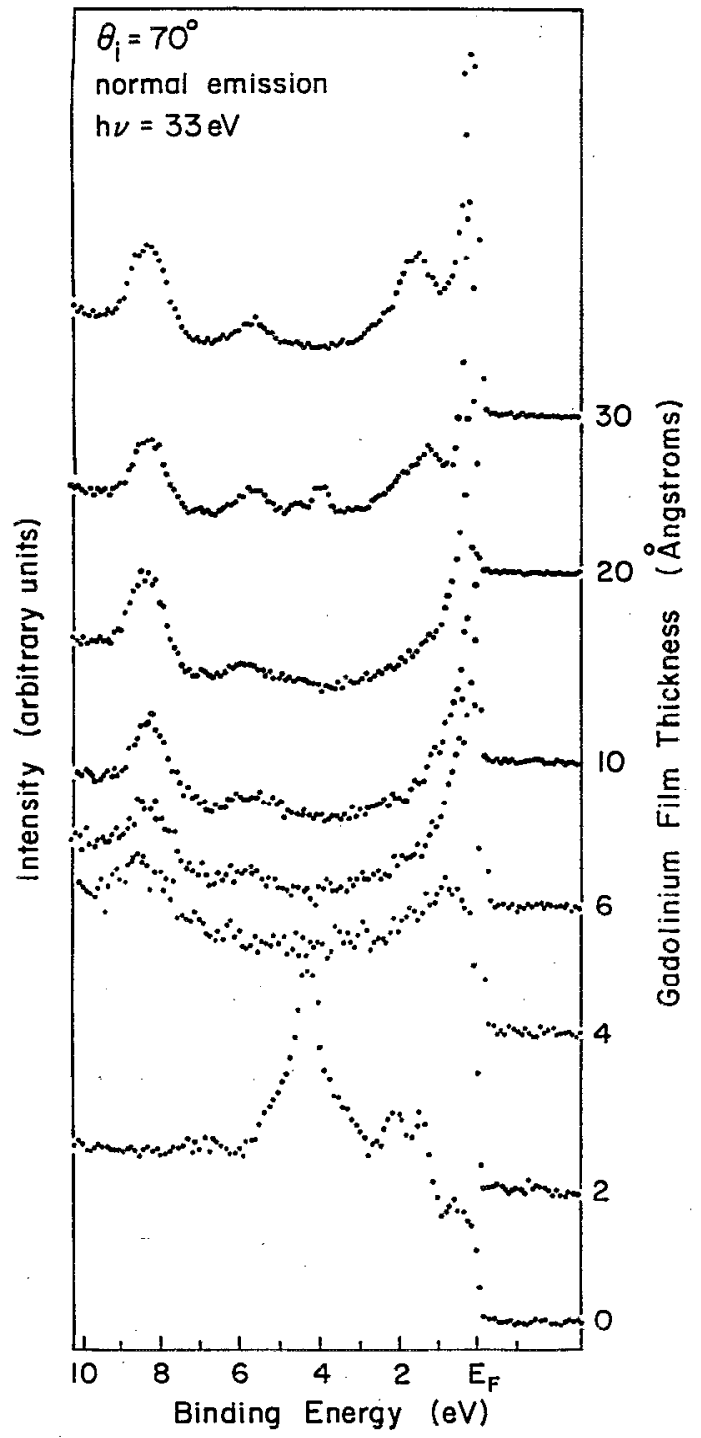

FIG. 1. Photoemission spectra of gadolinium overlayers on W(110). The photoelectrons were collected normal to the surface and the light is $p$ polarized so as to increase the relative signal of the surface state relative to the other bands.

The state at $0.2 \mathrm{eV}$ falls into a gap in the projected band structure at $\bar{\Gamma}^{\prime}$, based upon calculated ${ }^{1-6,8-9}$ and experimentally derived ${ }^{11}$ band structures for bulk gadolinium. This state shows conservation of two dimensionality of state, is independent of film thickncss, and is sensitive to the adsorption of oxygen and other contamination, as seen in Fig. 2. Since this state also has a very narrow full width at half-maximum, this state has been identified as a surface state. ${ }^{10}$ This state is predicted by a recently calculated band structure, ${ }^{9}$ which characterizes this state as a minority-spin magnetic surface state. This surface state does not fall into a gap of the projected tungsten density of states at $\overline{\bar{\Gamma}^{\prime}}$ and hybridization with the W(110) surface is possible, thus one cannot anticipate that this state will be as narrow for the thin films as one would expect for the thicker films, as is observed.

Another gadolinium band is increasingly distinct as a

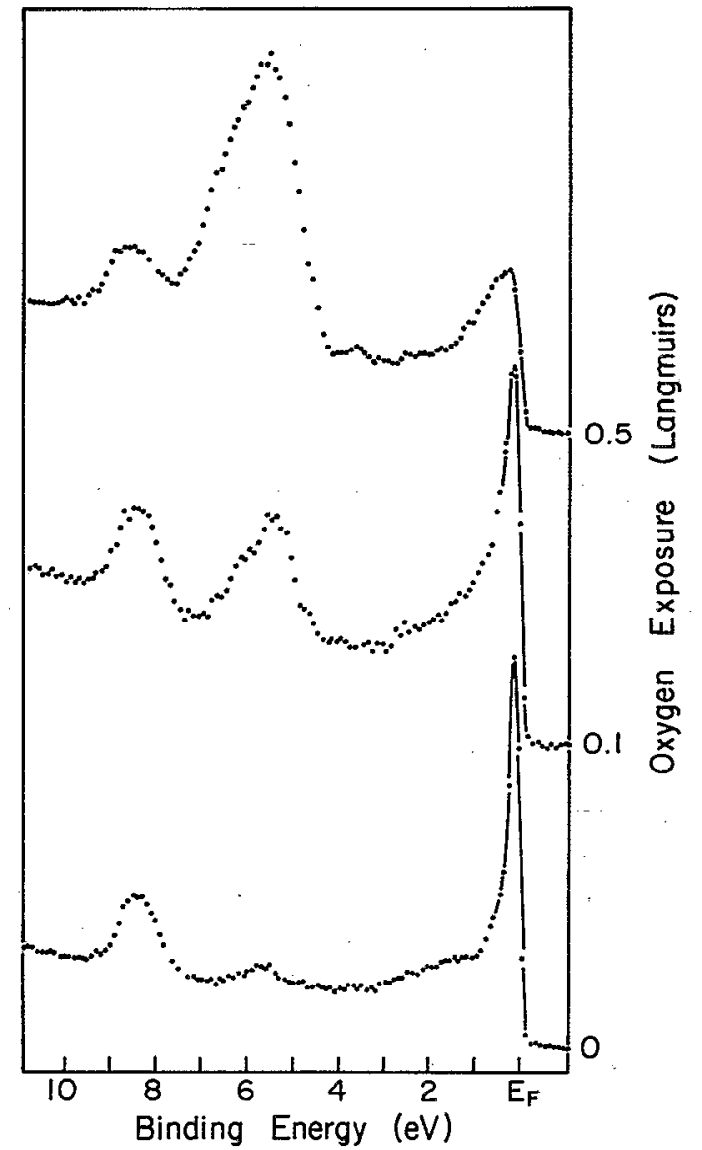

FIG. 2. Photoemission spectra of $15-\AA ̊ \AA$ gadolinium films on $W(110)$, following various oxygen exposures. The light incidence angle is $70^{\circ}(p$ polarization), and all photoelectrons were collected normal to the surface.

result of an increasing binding energy with increasing gadolinium coverage as seen in Fig. 1. There is increasing similarity of the bulk band-binding energies and oscillator strengths for the gadolinium overlayers on $W(110)$ and the gadolinium $5 d$ bulk bands ${ }^{11}$ with increasing film thickness. These gadolinium-induced states at $1-2 \mathrm{eV}$ below the Fermi energy have a binding energy that is sensitive to changes in photon energy, even for gadolinium films of 10 $\AA$. Sensitivity to changes in $\mathbf{k}_{\perp}$ (changes in photon energy) is characteristic of bulk bands, and the increasing resemblance of these states $1-2 \mathrm{eV}$ below $E_{F}$ to the bulk band structure at $\bar{\Gamma}^{\prime}$ (Ref. 11) with increasing film thickness is strongly indicative that these features are representative of the bulk band structure. It is important to realize that for the thinnest of gadolinium films, the lattice constant differs slightly from that of bulk gadolinium. ${ }^{16-18}$ This together with the additional complications of the two dimensionality of the film and hybridization of the gadolinium overlayer with the W(110) substrate results in the differences between the bulk band structure observed for the thicker films or by Himpsel and Reihl, ${ }^{11}$ and the band structure observed for the very thin gadolinium films.

With strong spin-orbit coupling the $5 p$ level separates into two $5 p_{1 / 2^{-}}$and $5 p_{3 / 2}$-based levels as has been observed at 28 and $21 \mathrm{eV}$, respectively (Refs. 12, 13, 27, and the 


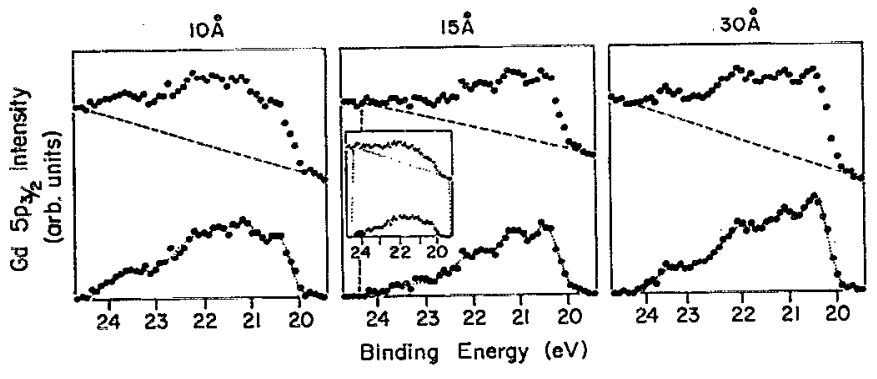

FIG. 3. Photoemission spectra across the gadolnium $5 p_{3 / 2}$ envelope for a $10-, 15-$, and $30-\AA$-thick gadolinium film on $W(110)$. Note the change in the relative multiplet oscillator strengths. This is particularly apparent after the photoemission background is removed. The insert shows the effect of one 1-L oxygen exposure on the 15- $\AA$-thick gadolinium film. The photon energy was $50 \mathrm{eV}$.

references therein). Fine structure can be observed within the $5 p$ levels, particularly within the $5 p_{3 / 2}$ envelope. We have recently identified this fine structure as a multiplet structure which is a result of $j-J$ coupling in the final state. ${ }^{27}$ The relatively weaker exchange interaction of the $5 p$ hole with the $4 f$ and $5 d$ electrons of gadolinium will lift the $J$ degeneracy. Neglecting the $5 d$ electrons for simplicity, the $j=\frac{3}{2}$ term of the hole (in the $5 p_{3 / 2}$ envelope) couples with the ${ }^{8} S_{7 / 2} 4 f$ levels (again neglecting the $5 d$ electrons) to form four terms of $\left(\frac{3}{2}, \frac{7}{2}\right)_{5},\left(\frac{3}{2}, \frac{7}{2}\right)_{4},\left(\frac{3}{2}, \frac{7}{2}\right)_{3}$, and $\left(\frac{3}{2}, \frac{7}{2}\right)_{2}$ (Ref. 27) as is observed at binding energies of 20.3 $\pm 0.1,20.9 \pm 0.1,22.0 \pm 0.2$, and $23.4 \pm 0.3 \mathrm{eV}$, respectively. Similar results, though not as pronounced, have also been obtained for terbium.

In Fig. 3, we present the $5 p_{3 / 2}$ photoemission feature as a function of gadolinium film thickness. There is a clear indication that the $\left(\frac{3}{2}, \frac{7}{2}\right)_{5}$ multiplet increases in oscillator strength relative to the other multiplets within the $5 p_{3 / 2}$ envelope with increasing gadolinium film thickness. Furthermore, with oxygen adsorption there is a pronounced change in the relative $5 p$ multiplet oscillator strengths as seen in the inset to Fig. 3. This can be correlated with the changes in the $5 d$ band structure as seen in Fig. 2.

The results in Fig. 3 indicate that the Gd $5 p\left(\frac{3}{2}, \frac{7}{2}\right)_{5}$ multiplet member, where $j$ for the $5 p_{3 / 2}$ hole is parallel to the $4 f$-derived $J$, is dramatically affected by oxidation and Gd film thickness. Oxidation destroys the gadolinium surface state (as seen in Fig. 2), while increasing film thickness leads to formation of a distinct surface state at $\bar{\Gamma}^{\prime}$. ${ }^{10}$ We do not have conclusive data to establish whether the Gd $\left(\frac{3}{2}, \frac{7}{2}\right)_{5}$ multiplet couples magnetically to the surface or bulk Gd $5 d$. We nonetheless postulate that the Gd $5 p$ to Gd $5 d$ final-state coupling (leading to the multiplet fine structure) is strongly influenced by the Gd $5 p$ surface-state magnetic ordering. Clearly there is a change in the $5 d$ band structure with increasing gadolinium film thickness while the occupied $4 f$ levels at $8.6 \mathrm{eV}$ binding energy are highly localized and exhibit little dependence upon film thickness as seen in Fig. 1. These results strongly support the postulate proposed elsewhere ${ }^{12-15}$ that photoemission from the $5 p$ levels is strongly influenced by final-state interactions with the $5 d$ levels, instead of solely a result of final-state interactions of the $4 f$ electrons with the $5 p$ hole.

In summary, we find that there are dramatic changes in the $5 d$ band structure with increasing film thickness. Some of these changes are associated with the development of a bulk band structure and a well-defined gadolinium surface state. These changes in the electronic band structure of gadolinium with increasing film thickness on $\mathrm{W}(110)$ also result in surprising changes to the photoemission features from the gadolinium core levels as a result of final-state coupling effects with the $5 d$ bands.

\section{ACKNOWLEDGMENTS}

This work was. funded by the U.S. DOE (Wisconsin and Syracuse) and the NSF (Texas). These studies were carried out both at the National Synchrotron Light Source at Brookhaven National Laboratory, which is funded by the DOE, and at the Synchrotron Radiation Center in Stoghton, Wisconsin, which is funded by the NSF.

${ }^{1}$ J. P. Dimmock and A. J. Freeman, Phys. Rev. Lett. 17, 750 (1964).

${ }^{2}$ R. E. Watson, A. J. Freeman, and J. P. Dimmock, Phys. Rev. 167, 497 (1968).

${ }^{3}$ B. N. Harmon and A. J. Freeman, Phys. Rev. B 10, 1979 (1974).

${ }^{4}$ B. N. Harmon, J. Phys. (Paris) Colloq. 40, C5-65 (1979).

${ }^{5}$ W. M. Temmerman and P. A. Sterne, J. Phys. Condens. Mat. 2, 5529 (1990).

${ }^{6} \mathrm{~J}$. Sticht and J. Kubler, Solid State Commun. 53, 529 (1985).

${ }^{7}$ W. Borgiel, G. Borstel, and W. Nolting, Solid State Commun. 60, 313 (1968).

${ }^{8}$ T. C. Leung, X. W. Wang, and B. N. Harmon, Physica B 149, 131 (1988).

${ }^{9}$ Ruqian Wu and A. J. Freeman, J. Magn. Magn. Mater. (in press).

${ }^{10}$ Dongqi Li, C. W. Hutchings, P. A. Dowben, C. Hwang; R.-T. Wu, M. Onellion, B. Andrews, and J. L. Erskine, J. Magn. Magn. Mater. (in press).

${ }^{11}$ F. J. Himpsel and B. Reihl, Phys. Rev. B 28, 574 (1983).

${ }^{12}$ P. A. Dowben, D. LaGraffe, and M. Onellion, J. Yhys. Condens. Mat. 1, 6571 (1989).

${ }^{13}$ D. LaGraffe, P. A. Dowben, and M. Onellion, Phys. Rev. B 10, 3348 (1989).

${ }^{14}$ D. LaGraffe, P. A. Dowben, and M. Onellion, Phys. Lett. A 147, 240 (1990).

${ }^{15}$ P. A. Dowben, D. LaGraffe, Dongqi Li, A. Miller, L. Zhang, L. Dottl, and M. Onellion, Phys. Rev. B 43, 3171 (1991).

16. Kolacziewicz and E. Bauer, Surf. Sci. 175, 487 (1986):

${ }^{17}$ D. Weller, S. F. Alvarado, M. Campagna; W. Gudat, and D. D. Sarma, J. Less-Common Met. 111, 277 (1985).

${ }^{18}$ D. Weller and S. F. Alvarado, J. Appl. Phys. 59, 2908 (1986)

${ }^{19}$ A. Ciszewski and A. J. Melmed, Surf. Sci. 145, L471 (1984).

${ }^{20}$ D. Weller and S. F. Alvarado, Z. Phys. B 58, 261 (1985).

${ }^{21}$ D. Weller, S. F. Alvarado, W. Gudat, K. Schroder, and M. Campagna, Phys. Rev. Lett. 54, 1555 (1985).

${ }^{22}$ K. Babersche, M. Farle, and M. Zomak, Appl. Phys. A 44, 13 (1987)

${ }^{23}$ M. Farle, A. Berhaus, and K. Baberschke, Phys. Rev. B 39, 4838 (1989).

${ }^{24}$ M. Farle and K. Baberschke, Phys. Rev. Lett. 58, 511 (1987).

${ }^{25}$ E. Jensen and D. M. Wielisczka, Phys. Rev. B 30, 7340 (1984).

${ }^{26}$ S. C. Wu, H. Li, D. Tian, J. Quinn, Y. S. Li, F. Jona, J. Sokolov, and N. E. Christensen, Phys. Rev. B 41, 11911 (1990).

${ }^{27}$ Dongqi Li, P. A. Dowben, and M. Onellion, Mater. Res. Soc. Symp. Proc. (in press). 